Part of Journal of Research of the National Bureau of Standards, Volume 26, June 1941

\title{
COMPARATIVE TESTS OF CHEMICAL GLASSWARE
}

\author{
By Edward Wichers, Alfred N. Finn, and W. Stanley Clabaugh
}

\section{ABSTRACT}

This paper describes the results of comparative tests of four brands of chemical glassware: Tamworth-Glasbake, Kimble No. N51a, chemical Pyrex, and Vycor (Corning Glass Works' 96-percent silica glass No. 790). The wares were compared with respect to resistance to acid, alkaline, and nearly neutral reagents, and resistance to thermal and mechanical shock. The chemical composition and the thermal expansivity of each glass were also determined.

\section{CONTENTS}

I. Introduction .

II. Chemical composition

III. Thermal expansivity _..._.

IV. Relative chemical resistance _._.

1. Acid reagents and water

2. Alkaline reagents

3. Nearly neutral reagents

V. Resistance to mechanical shock

VI. Resistance to thermal shock

\section{INTRODUCTION}

Three brands of borosilicate glass, varying somewhat in composition and general properties, are now produced in the United States in the form of beakers, flasks, and other apparatus for general laboratory use. These three wares are chemical Pyrex (Corning Glass Works' No. 774); Kimble Glass Co.'s No. N51a; and TamworthGlasbake. ${ }^{1}$

A series of tests designed to determine the relative resistance to chemical attack of these three wares has been made at the National Bureau of Standards, similar to tests made about 25 years ago, ${ }^{2}$ when the European wares commonly used up to that time began to be displaced by several products of American manufacturers. The chemical compositions of the glasses, and their thermal coefficients of expansion, were also determined, and some information gained concerning their resistance to mechanical and thermal shock.

\footnotetext{
1 The ware bearing this mark is manufactured by the McKee Glass Co., Jeannette, Pa., and distributed by Tamworth Associates, Needham Heights, Mass. The McKee Glass Co. has stated that ware of the same composition will be distributed in certain parts of the country under the mark "Glasbake."

2 Percy H. Walker and F. W. Smither, Comparative tests of chemical glassware, Tech. Pap. BS 10, (1918) T107; J. Ind. Eng. Chem. 9, 1090 (1917).
} 
A new type of chemical glassware sold under the trademark Vycor (Corning Glass Works' 96-percent silica glass No. 790) was included in some of the tests.

Erlenmeyer flasks of $250-\mathrm{ml}$ capacity were used for nearly all the tests described in this report. The factories where the different glasses are produced were visited by one of the authors to see the flasks made (except the Vycor) and to earmark them for delivery to the Bureau. At the same time, samples of the glasses in the form of rods were prepared and brought to the Bureau. These samples, together with some in the form of slabs, were used for some of the measurements of the thermal expansivity.

\section{CHEMICAL COMPOSITION}

The major constituents of the glasses tested were determined by routine chemical analyses. ${ }^{3}$ The results obtained, as given in table 1 , are intended to show the approximate composition, except with respect to arsenic and antimony. These elements were determined with greater care. ${ }^{4}$

TABLE 1.-Composition of the samples

\begin{tabular}{|c|c|c|c|c|}
\hline Constituent & Glasbake & Kimble & Pyrex & Vycor d \\
\hline 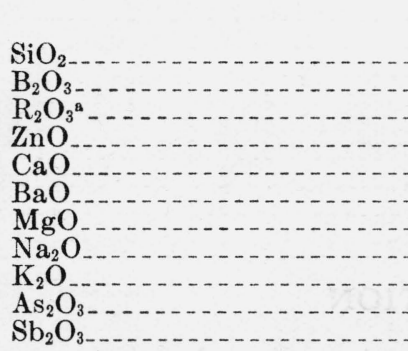 & $\begin{array}{r}\text { Percent } \\
78.4 \\
14.0 \\
2.5 \\
\text { n. d. } \\
0.1 \\
\text { n. d. } \\
\text { neg. } \\
5.0 \\
\text { neg. } \\
0.037 \\
.038\end{array}$ & $\begin{array}{r}\text { Percent } \\
74.7 \\
9.6 \\
5.6 \\
0.1 \\
.9 \\
2.2 \\
\text { neg. } \\
6.4 \\
0.5 \\
.027 \\
.009\end{array}$ & $\begin{array}{r}\text { Percent } \\
81.0 \\
\text { 13. } 0 \\
2.2 \\
\text { n. d. } \\
\text { neg. } \\
\text { n. d. } \\
\text { n. d. } \\
3.6 \\
0.2 \\
.002 \\
\text { n. d. }\end{array}$ & $\begin{array}{r}\text { Percent } \\
96.3 \\
2.9 \\
0.4 \\
\text { n. d. } \\
\text { neg. } \\
\text { n. d. } \\
\text { n. d. } \\
<0.02 \\
<.02 \\
.005 \\
\text { n. d. }\end{array}$ \\
\hline
\end{tabular}

- Chiefly $\mathrm{Al}_{2} \mathrm{O}_{3}$.

b "neg." indicates a negligible amount of the constituent.

- "n. d." indicates the corresponding constituent was not detected.

d 0.3 percent of undetermined constituents.

\section{THERMAL EXPANSIVITY}

The average coefficient of linear expansion in the range $20^{\circ}$ to $300^{\circ} \mathrm{C}$ was determined with the apparatus described by Souder and Hidnert ${ }^{5}$ on specimens of the rods obtained for that purpose. The coefficient was also determined by the interferometer method described by Peters and Cragoe, ${ }^{6}$ as amplified by Saunders, ${ }^{7}$ on specimens cut from slabs and from flasks. The rods were tested first "as received" (i. e., air-cooled or unannealed), and again after the same specimens

\footnotetext{
3 Analyses made by Francis W. Glaze.

- Determinations made by J. A. Scherrer.

- Wilmer Souder and Peter Hidnert, Measurement on the thermal expansion of fused silica, BS Sci. Pap. 21, 1 (1926) S524. The measurements by this method were made by Hidnert.

$\therefore$ C. G. Peters and C. H. Cragoe, Measurements on the thermal dilatation of glasse sat high temperatures, BS Sci. Pap. 16, 449 (1920) S393.

7 James B. Saunders, Improved interferometric procedure with application to expansion measurements, J. Research NBS 23, 179 (1939) RP1227. The measurements by this method were made by L. H. Maxwell and by Saunders.
} 
were carefully annealed. The annealing consisted in heating the rods to $590^{\circ} \mathrm{C}$ for 5 hours, cooling to $515^{\circ} \mathrm{C}$ at the rate of $8^{\circ} \mathrm{C}$ per hour, holding at the latter temperature for 48 hours, and then cooling slowly to room temperature. The initial cooling rate from $515^{\circ} \mathrm{C}$ was about $1^{\circ} \mathrm{C}$ per hour and was gradually increased to a maximum of $10^{\circ} \mathrm{C}$ per hour as the temperature fell. ${ }^{8}$

The results of the expansivity measurements are given in table 2 . The data show that the two methods of measurement give practically identical results, unless there were compensating differences in the samples and methods used.

TABLE 2.-Average coefficient of linear expansion $\times 10^{\circ}$, between $20^{\circ}$ and $300^{\circ} \mathrm{C}$

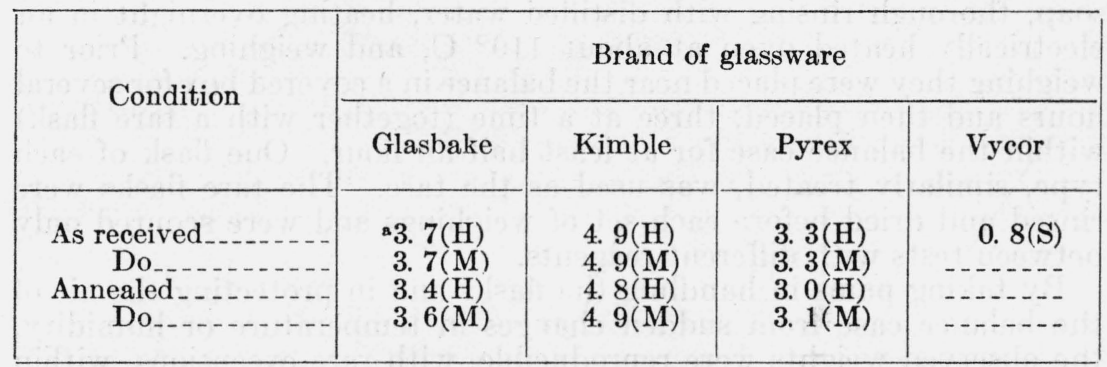

- Values followed by (H) were obtained by P. Hidnert, those followed by (M) by L. H. Maxwell, and that followed by (S) by J. B. Saunders.

\section{RELATIVE CHEMICAL RESISTANCE}

In studying the effect of various aqueous solutions and other reagents on the glasses, no attempt was made to determine the nature of the reactions, that is, to find out what changes occurred at the surface of the glass or whether the material removed was of the same composition as the glass. An exception to this general rule was made in determining the amount of arsenic removed by alkaline solutions, since this is of known interest in certain uses of chemical glassware, such as in the determination of arsenic in insecticide residues on fruits. These results appear in table 5 , footnote a.

In general, it appeared that the analyst's interest would lie in the total amount of substance removed from the glass, in relation both to the contamination of solutions undergoing analysis, and to the relative rates at which the wares would become unserviceable through roughening of the surface. Because precipitates tend to adhere to etched glass surfaces so tenaciously as to make quantitative transfers difficult or impossible, the useful life of much chemical glassware may be ended by excessive etching or scratching of the inner surfaces, rather than by breakage.

Since the chemist cannot readily have glassware made to order (with respect to composition or properties) but must necessarily make a choice among products that are commercially available, it seemed useless to attempt to devise tests that might yield results which would be independently reproducible and which might have some absolute significance. Instead, the methods of testing chemical resistance were

\footnotetext{
${ }^{8}$ It happened to be convenient to anneal the rods in the same furnace with some optical glass-hence this unnecessarily long schedule.
} 
planned solely to yield comparative results. The three brands (all except the Vycor) were always treated simultaneously, thus making it unnecessary to define the conditions of treatment very closely. All of the tests of chemical resistance were made on samples taken from a single lot of 250-ml Erlenmeyer flasks of each type of ware. It is possible that similar tests made on other lots of the wares and in other laboratories would not yield the same numerical results as those reported here, but it is believed that, barring significant changes of composition in any of the wares, similar tests would give the same relative results.

All the flasks used were numbered for identification. They were prepared for testing by gentle scouring, inside and out, with a pumice soap, thorough rinsing with distilled water, heating overnight in an electrically heated oven at about $110^{\circ} \mathrm{C}$, and weighing. Prior to weighing they were placed near the balance in a covered box for several hours and then placed, three at a time (together with a tare flask) within the balance case for at least half an hour. One flask of each type, similarly treated, was used as the tare. The tare flasks were rinsed and dried before each set of weighings and were scoured only between tests with different reagents.

By taking pains in handling the flasks and in protecting the air of the balance case from sudden changes in temperature or humidity, the observed weights were reproducible, with rare exceptions, within 0.1 or $0.2 \mathrm{mg}$. Losses in weight caused by scouring and rinsing the flasks were negligible. These conclusions were reached from a series of repeated weighings of flasks not treated, except by cleaning and drying, which are shown in table 3 . In this series the tare flasks were not scoured, but only rinsed and dried whenever the others were scoured, rinsed, and dried. 
TABLE 3.-Repeated weighings of flasks to determine precision of weighing

\begin{tabular}{|c|c|c|c|c|c|c|c|c|c|c|}
\hline \multirow{2}{*}{ Treatment } & \multirow{2}{*}{$\begin{array}{c}\text { Relative } \\
\text { humidity }\end{array}$} & \multicolumn{3}{|c|}{ Glasbake b } & \multicolumn{3}{|c|}{ Kimble • } & \multicolumn{3}{|c|}{ Pyrex d } \\
\hline & & No. 13 & No. 14 & No. 15 & No. 22 & No. 23 & No. 24 & No. 15 & No. 16 & No. 17 \\
\hline $\begin{array}{l}\text { Scoured, etc } \\
\text { Do } \\
\text { Reweighed after standing } 3 \mathrm{hr} \\
\text { Reweighed after standing over- }\end{array}$ & $\begin{array}{r}\% \\
6 \text { to } 11 \\
14 \\
14\end{array}$ & $\begin{array}{l}\text { 16. } 5285 \\
\text { 16. } 5285 \\
16.5285\end{array}$ & $\begin{array}{l}\text { 13. } 6856 \\
\text { 13. } 6858 \\
\text { 13. } 6857\end{array}$ & $\begin{array}{l}\text { 2. } 7927 \\
\text { 2. } 7927 \\
\text { 2. } 7928\end{array}$ & $\begin{array}{l}\text { 2. } 2975 \\
\text { 2. } 2976 \\
\text { 2. } 2975\end{array}$ & $\begin{array}{l}\text { 2. } \\
\text { 27775 } \\
\text { 2. } 4777 \\
\text { 2. } 4775\end{array}$ & $\begin{array}{l}\text { 5 } \\
\text { 5. } \\
\text { 5. } 8355 \\
\text { 5. } 8355\end{array}$ & $\begin{array}{r}0 . \\
0991 \\
.6990 \\
.6990\end{array}$ & $\begin{array}{l}\text { 7. } 1825 \\
\text { 7. } 1825 \\
\text { 7. } 1825\end{array}$ & $\begin{array}{l}\text { 5. } 0996 \\
5.0995 \\
5.0995\end{array}$ \\
\hline $\begin{array}{l}\text { night, } \\
\text { Scoured, etc.... }\end{array}$ & $\begin{array}{r}16 \text { to } 17 \\
34 \text { tก } 38\end{array}$ & $\begin{array}{l}\text { 16. } 5285 \\
16.5285\end{array}$ & $\begin{array}{l}\text { 13. } 6858 \\
\text { 13. } 6860\end{array}$ & $\begin{array}{l}\text { 2. } 7926 \\
\text { 2. } 7928\end{array}$ & $\begin{array}{l}\text { 2. } 2975 \\
\text { 2. } 2976\end{array}$ & $\begin{array}{l}\text { 2. } 4775 \\
\text { 2. } 4776\end{array}$ & $\begin{array}{l}\text { 5. } 8355 \\
\text { 5. } 8355\end{array}$ & $\begin{array}{r}.6990 \\
.6990\end{array}$ & $\begin{array}{l}\text { 7. } 1825 \\
\text { 7. } 1822\end{array}$ & $\begin{array}{l}5.0995 \\
5.0996\end{array}$ \\
\hline
\end{tabular}

- The weights are the difference between each flask and the corresponding tare flask.

b The weight of the tare flask was $78 \mathrm{~g}$.

The weight of the tare flask was $58 \mathrm{~g}$.
d The weight of the tare flask was $76 \mathrm{~g}$. 
With a few exceptions, which will be indicated, $200 \mathrm{ml}$ of the selected reagent was placed in each of three flasks of each of the wares, which were heated simultaneously on an electric hot plate. Gentle boiling was maintained for 6 hours. During the treatment, each flask was closed with a funnel-shaped cover, made of the same glass, to serve as a partial condenser. Flattened pellets of gold sponge served admirably to prevent bumping and were not attacked by any of the reagents except perchloric acid, which did attack them slightly. About midway of the 6-hour period of boiling, the volume of reagent was restored to about $200 \mathrm{ml}$ by replenishing with hot water, except as otherwise noted. The flasks made of the different glasses were so nearly of the same shape and capacity that variations in the surface exposed to attack were regarded as negligible. Because they were exposed to condensing vapors and to spray from the boiling liquid, the glass surfaces above the level of the reagent were also attacked, although probably not equally with the submerged portions.

After the 6-hour treatment, the flasks were emptied, rinsed with water, gently scrubbed inside and out, then thoroughly rinsed again, and heated in the oven overnight at about $110^{\circ} \mathrm{C}$. The weighing procedure after treatment was the same as before. The same flasks were then subjected to a second treatment for 6 hours, and in some instances to a third.

The Vycor flasks were not received until after the tests had been started. The tests of this ware were, therefore, not made simultaneously with those of any of the other three brands. However, since the conditions of test were duplicated rather closely, it is believed that the results can be safely compared with those for the other wares.

The reagents used, besides distilled water, can be divided into three groups: Strongly acid, strongly alkaline, and nearly neutral. The results obtained with the three groups of reagents are shown in tables 4,5 , and 6 . For convenience, the results obtained with water are included with those of the acid group.

In order to show the extent of the variations in results obtained with different flasks, and during the successive periods of test, all of the individual results are tabulated, together with the averages for each flask and for each period of test. All the results obtained are reported. Only two results (for Vycor flasks treated with water) have been rejected in computing averages. These two were rejected because they deviate excessively from the average of the others in the same series.

Since the average deviation of the 45 trial weighings (from the averages of each of the nine groups of five weighings shown in table 3 ) is $0.05 \mathrm{mg}$, and since two weighings are involved in each test of a flask, differences in individual results in tables 4,5 , and 6 , greater than 0.1 $\mathrm{mg}$ can be regarded as significant, in general. However, since the experimental work was not planned specifically with the object of detecting differences in chemical resistance between individual specimens of glassware, it is believed that more attention should be given to the average results for each group of flasks. Again, although significant differences seem to occur in some instances between the results for the successive periods of treatment, their failure to occur more generally makes them hard to explain. In the graphic presentation of the results, in figures 1,2 , and 3, only the grand averages are shown.

In comparing results obtained with different reagents, account should be taken of the occasional variations in the testing procedure or in the length of the periods of test. 


\section{ACID REAGENTS AND WATER}

The numbers of the following explanatory notes correspond to the numbers attached to each group of data in table 4 and figure 1:

1. Distilled water.

2. Approximately normal sulfuric acid.

3. Approximately normal phosphoric acid (one-third molar).

4. Approximately $6 N$ ("constant-boiling") hydrochloric acid. About midway of the 6-hour period of boiling the flasks were replenished with acid of the same strength instead of with water.

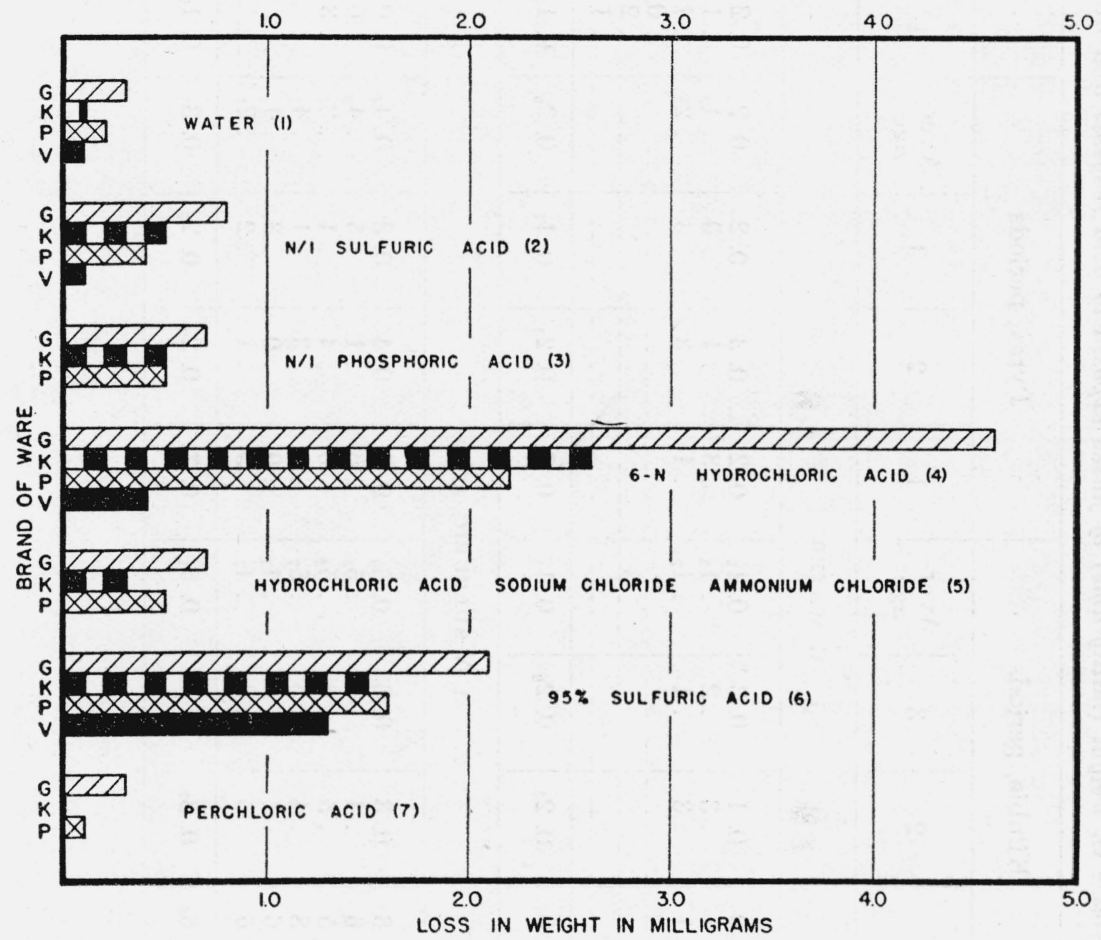

FIGURE 1.-Comparative resistance to acid reagents and water.

A verage loss in weight, in milligrams, per flask per 6-hour period of exposure.

5. A solution containing $50 \mathrm{ml}$ of concentrated hydrochloric acid, $50 \mathrm{~g}$ of sodium chloride, and $50 \mathrm{~g}$ of ammonium chloride, in 1 liter.

6. Sulfuric acid, 95 percent. Fifty $\mathrm{ml}$ was used instead of the usual $200 \mathrm{ml}$, and the flasks were heated on a gas hot plate. Since the area of glass exposed to the boiling acid was not the same as with most of the other reagents, the results are not directly comparable with those obtained with the others. No replenishment of acid was necessary.

7. Perchloric acid. Fifty $\mathrm{ml}$ of 60-percent acid was used for the first period of 6 hours. The electric hot plate used did not supply enough heat to keep the acid boiling, but much of the acid evaporated. For the second period, $100 \mathrm{ml}$ of acid was used. The flasks were heated on a gas hot plate to keep the acid boiling gently. No acid was added during either of the 6 -hour periods. 


\begin{tabular}{|c|c|c|c|c|c|c|c|c|c|c|c|c|c|c|c|}
\hline \multicolumn{4}{|c|}{ Glasbake, periods } & \multicolumn{4}{|c|}{ Kimble, periods } & \multicolumn{4}{|c|}{ Pyrex, periods } & \multicolumn{4}{|c|}{ Vycor, periods } \\
\hline 1 & 2 & 3 & $\begin{array}{c}\text { Aver- } \\
\text { age }\end{array}$ & 1 & 2 & 3 & $\begin{array}{c}\text { Aver- } \\
\text { age }\end{array}$ & 1 & 2 & 3 & $\begin{array}{c}\text { Aver- } \\
\text { age }\end{array}$ & 1 & 2 & 3 & $\begin{array}{l}\text { Aver- } \\
\text { age }\end{array}$ \\
\hline
\end{tabular}

1. WATER

\begin{tabular}{|c|c|c|c|c|c|c|c|c|c|c|c|c|c|c|c|}
\hline $\begin{array}{r}0.3 \\
.5 \\
.3 \\
\\
\\
\end{array}$ & \begin{tabular}{r}
0.5 \\
.5 \\
.5 \\
- \\
\hdashline \\
\end{tabular} & $\begin{array}{r}0.0 \\
0 \\
.5 \\
-5 \\
\\
\end{array}$ & \begin{tabular}{r}
$0.2_{7}$ \\
$.3_{3}$ \\
$.4_{3}$ \\
\hdashline \\
\end{tabular} & $\begin{array}{r}0.0 \\
+.1 \\
.1 \\
\\
\hdashline\end{array}$ & \begin{tabular}{c}
0.1 \\
.3 \\
.3 \\
\hdashline \\
\hdashline-0 \\
\end{tabular} & \begin{tabular}{c}
0.3 \\
.2 \\
.1 \\
\hdashline \\
\hdashline
\end{tabular} & $\begin{array}{r}0.1_{3} \\
.1_{3} \\
.1_{7} \\
\hdashline \\
\hdashline\end{array}$ & $\begin{array}{r}0.2 \\
.3 \\
.1 \\
\hdashline \\
\hdashline\end{array}$ & $\begin{array}{r}0.3 \\
.1 \\
.3 \\
\\
\end{array}$ & \begin{tabular}{c}
0.2 \\
.0 \\
.3 \\
- \\
\hdashline \\
\hdashline
\end{tabular} & $\begin{array}{r}0.2_{3} \\
\cdot 1_{3} \\
.2_{3} \\
\hdashline \\
\hdashline\end{array}$ & $\begin{array}{r}0.2 \\
.1 \\
.4 \\
.0 \\
.2 \\
.1\end{array}$ & $\begin{array}{r}\text { b } 0.9 \\
\text { b1. } 0 \\
0.5 \\
.0 \\
.0 \\
.0\end{array}$ & \begin{tabular}{c}
0.2 \\
.2 \\
.0 \\
\hdashline \\
\hdashline \\
\hdashline
\end{tabular} & $\begin{array}{r}0.2_{0} \\
.1_{5} \\
.3_{0} \\
.0_{0} \\
.1_{0} \\
.05\end{array}$ \\
\hline Avg $\ldots-3_{7}$ & $0.5_{0}$ & $0.1_{7}$ & $0.3_{4}$ & $0.0_{0}$ & 0. $2_{3}$ & 0. $2_{0}$ & $0.1_{4}$ & 0. $2_{0}$ & $0.2_{3}$ & 0. $1_{7}$ & $0.2_{0}$ & 0. $1_{7}$ & 0. $1_{2}$ & $0.1_{3}$ & $0.1_{4}$ \\
\hline
\end{tabular}

2. $1 N$ SULFURIC ACID

\begin{tabular}{|c|c|c|c|c|c|c|c|c|c|c|c|c|c|c|c|}
\hline $\begin{array}{r}1.1 \\
1.1 \\
1.1 \\
1.3 \\
0.9 \\
.8\end{array}$ & $\begin{array}{r}0.9 \\
1.1 \\
0.4 \\
.7 \\
.3 \\
.7\end{array}$ & $\begin{array}{r}0.9 \\
1.0 \\
0.8 \\
.8 \\
.9 \\
.2\end{array}$ & $\begin{array}{r}\text { 0. } 9_{7} \\
\text { 1. } 0_{7} \\
\text { 0. } 7_{7} \\
.9_{3} \\
.7_{0} \\
.5_{7}\end{array}$ & $\begin{array}{r}0.6 \\
.6 \\
.5 \\
.8 \\
.6 \\
.6\end{array}$ & $\begin{array}{r}0.3 \\
.4 \\
.5 \\
.4 \\
.1 \\
.5\end{array}$ & $\begin{array}{r}0.6 \\
.6 \\
.6 \\
.5 \\
.6 \\
.3\end{array}$ & $\begin{array}{r}0.5_{0} \\
.5_{3} \\
.5_{3} \\
.5_{7} \\
.4_{3} \\
.4_{7}\end{array}$ & $\begin{array}{l}0.7 \\
.6 \\
.6 \\
.9 \\
.9 \\
.5\end{array}$ & $\begin{array}{r}0.4 \\
.1 \\
.4 \\
.3 \\
.0 \\
.1\end{array}$ & $\begin{array}{r}0.3 \\
.5 \\
.1 \\
.1 \\
.3 \\
.2\end{array}$ & $\begin{array}{r}0.4_{7} \\
.4_{0} \\
.3_{7} \\
.4_{3} \\
.4_{0} \\
.27\end{array}$ & \begin{tabular}{c}
0.0 \\
0 \\
.3 \\
-3 \\
\hdashline- \\
\hdashline
\end{tabular} & \begin{tabular}{c}
0.2 \\
.2 \\
+.1 \\
\hdashline.-- \\
\hdashline..- \\
-1
\end{tabular} & 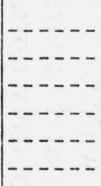 & $\begin{array}{r}0.1_{0} \\
.1_{0} \\
.1_{0}\end{array}$ \\
\hline vg $\ldots 1.0_{5}$ & 0. $6_{8}$ & 0. $7_{7}$ & 0. $8_{3}$ & $0.6_{2}$ & 0. $3_{7}$ & $0.5_{3}$ & $0.5_{1}$ & 0. $7_{0}$ & 0. $2_{2}$ & 0. $2_{5}$ & 0. 39 & $0.1_{0}$ & 0. $1_{0}$ & -- & $0.1_{0}$ \\
\hline
\end{tabular}


3. $1 N$ PHOSPHORIC ACID

\begin{tabular}{|c|c|c|c|c|c|c|c|c|c|c|c|c|c|c|c|}
\hline $\begin{array}{r}0.9 \\
.9 \\
.9 \\
\end{array}$ & $\begin{array}{r}0.5 \\
.4 \\
.5 \\
\end{array}$ & 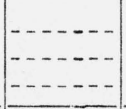 & $\begin{array}{r}0.7_{0} \\
.6_{5} \\
.7_{0} \\
\end{array}$ & $\begin{array}{r}0.7 \\
.3 \\
.7 \\
\end{array}$ & $\begin{array}{r}0.5 \\
.3 \\
.6 \\
\end{array}$ & 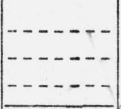 & $\begin{array}{r}0.6_{0} \\
.3_{0} \\
.6_{5} \\
\end{array}$ & $\begin{array}{r}0.6 \\
.7 \\
.6\end{array}$ & $\begin{array}{r}0.4 \\
.2 \\
.5\end{array}$ & 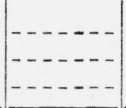 & $\begin{array}{r}0.5_{0} \\
.4_{5} \\
.5_{5}\end{array}$ & $\mid \begin{array}{c}-\cdots \\
---\cdots \\
---\cdots\end{array}$ & $\mid \begin{array}{l}-\cdots \\
-\cdots \\
-\cdots\end{array}$ & $\mid \begin{array}{l}-\cdots \\
-\cdots--- \\
-\cdots---\end{array}$ & 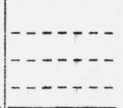 \\
\hline Avg $\ldots . .0 .90$ & 0.47 & $-\cdots+$. & $0.6_{8}$ & $0.5_{7}$ & 0.47 & 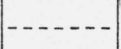 & $0.5_{2}$ & 0. $6_{3}$ & $0.3_{7}$ & 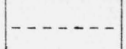 & $0.5_{0}$ & $-\cdots--$ & $-\cdots$ & -- & 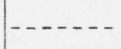 \\
\hline \multicolumn{16}{|c|}{ 4. CONSTANT-BOILING HYDROCHLORIC ACID } \\
\hline $\begin{array}{l}\text { 4. } 1 \\
\text { 4. } 7 \\
\text { 4. } 1\end{array}$ & $\begin{array}{l}5.0 \\
4.8 \\
4.7\end{array}$ & 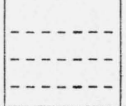 & $\begin{array}{l}\text { 4. } 5_{5} \\
\text { 4. } 7_{5} \\
\text { 4. } 4_{0}\end{array}$ & $\begin{array}{l}2.4 \\
2.5 \\
2.3\end{array}$ & $\begin{array}{l}2.9 \\
2.5 \\
2.8\end{array}$ & 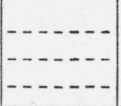 & $\begin{array}{l}2.6_{5} \\
2.5_{0} \\
2.5_{5}\end{array}$ & $\begin{array}{l}2.3 \\
2.1 \\
\text { 2. } 4\end{array}$ & $\begin{array}{l}2.3 \\
2.2 \\
2.0\end{array}$ & 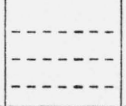 & $\begin{array}{l}\text { 2. } 3_{0} \\
\text { 2. } 1_{5} \\
\text { 2. } 2_{0}\end{array}$ & $\begin{array}{r}0.5 \\
.5 \\
.6\end{array}$ & $\begin{array}{r}0.2 \\
.3 \\
.3\end{array}$ & $\mid \begin{array}{c}-\cdots \\
-\cdots-- \\
-\cdots---\end{array}$ & $\begin{array}{r}0.3_{5} \\
.4_{0} \\
.4_{5}\end{array}$ \\
\hline Avg. . $4.3_{0}$ & 4. $8_{3}$ & 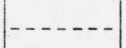 & 4. $5_{7}$ & 2. $4_{0}$ & 2. $7_{3}$ & $-\cdots---$ & 2. $5_{7}$ & 2. 27 & 2. $1_{7}$ & ----- & 2. $2_{2}$ & $0.5_{3}$ & 0. $2_{7}$ & $---\cdot-$ & 0. $4_{0}$ \\
\hline
\end{tabular}

5. HYDROCHLORIC ACID-SODIUM CHLORIDE-AMMONIUM CHLORIDE

\begin{tabular}{|c|c|c|c|c|c|c|c|c|c|c|c|c|c|c|c|}
\hline $\begin{array}{l}0.7 \\
\text { 1. } 1 \\
0.7\end{array}$ & $\begin{array}{r}0.7 \\
.4 \\
.7\end{array}$ & 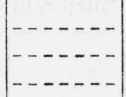 & $\begin{array}{r}0.7_{0} \\
.7_{5} \\
.7_{0}\end{array}$ & $\begin{array}{r}0.6 \\
.2 \\
.4\end{array}$ & $\begin{array}{r}0.3 \\
.2 \\
.3\end{array}$ & 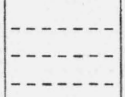 & $\begin{array}{r}0.4_{5} \\
.2_{0} \\
.3_{5}\end{array}$ & $\begin{array}{l}0.5 \\
1.0 \\
0.6\end{array}$ & $\begin{array}{r}0.2 \\
.2 \\
.3\end{array}$ & 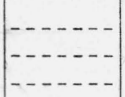 & $\begin{array}{r}0.3_{5} \\
.6_{0} \\
.4_{5}\end{array}$ & $\mid \begin{array}{c}----- \\
-\cdots--- \\
-\cdots---\end{array}$ & $\mid \begin{array}{l}-\cdots \\
-\cdots---- \\
-\cdots--\cdots\end{array}$ & $\mid \begin{array}{l}---- \\
--\cdots-- \\
--\cdots--\end{array}$ & $\mid \begin{array}{l}- \\
-\cdots \\
-\cdots\end{array}$ \\
\hline Avg $\ldots .8_{3}$ & $0.6_{0}$ & $\ldots \ldots$ & $0.7_{2}$ & $0.4_{0}$ & $0.2_{7}$ & $-\ldots . .--$ & $0.3_{3}$ & $0.7_{0}$ & $0.2_{3}$ & $\ldots \ldots$ & $0.4_{7}$ & $\cdots$ & & $\ldots$ & $\ldots$ \\
\hline
\end{tabular}

6. CONCENTRATED SULFURIC ACID

\begin{tabular}{|c|c|c|c|c|c|c|c|c|c|c|c|c|c|c|c|}
\hline $\begin{array}{l}\text { 1. } 1 \\
\text { 1. } 1 \\
\text { 2. } 1\end{array}$ & $\begin{array}{l}\text { 1. } 3 \\
\text { 2. } 1 \\
\text { 4. } 9\end{array}$ & $\begin{array}{l}\text { 1. } 6 \\
\text { 1. } 0 \\
\text { 4. } 1\end{array}$ & $\begin{array}{l}\text { 1. } 3_{3} \\
\text { 1. } 4_{0} \\
\text { 3. } 7_{0}\end{array}$ & $\begin{array}{l}\text { 1. } 0 \\
\text { 1. } 0 \\
\text { 1. } 0\end{array}$ & $\begin{array}{l}0.9 \\
\text { 1. } 6 \\
\text { 3. } 2\end{array}$ & $\begin{array}{l}1.9 \\
\text { 1. } 3 \\
\text { 1. } 3\end{array}$ & $\begin{array}{l}\text { 1. } 2_{7} \\
\text { 1. } 3_{0} \\
1.8_{3}\end{array}$ & $\begin{array}{l}1.2 \\
0.8 \\
1.5\end{array}$ & $\begin{array}{l}2.8 \\
2.2 \\
\text { 2. } 0\end{array}$ & $\begin{array}{l}\text { 1. } 1 \\
\text { 1. } 0 \\
\text { 2. } 2\end{array}$ & $\begin{array}{l}\text { 1. } 7_{0} \\
\text { 1. } 3_{3} \\
\text { 1. } 9_{0}\end{array}$ & $\begin{array}{r}0.9 \\
.9 \\
1.1\end{array}$ & $\begin{array}{l}\text { 1. } 1 \\
\text { 2. } 0 \\
\text { 1. } 0\end{array}$ & $\begin{array}{l}1.4 \\
1.5 \\
1.5\end{array}$ & $\begin{array}{r}\text { '1. } 1_{3} \\
\text { 1. } 4_{7} \\
\text { 1. } 2_{0}\end{array}$ \\
\hline Avg $\ldots .-1.4_{3}$ & 2. $7_{7}$ & 2. $2_{3}$ & 2. $1_{4}$ & 1. $0_{0}$ & 1. $9_{0}$ & 1. $5_{0}$ & 1. $4_{7}$ & 1. $1_{7}$ & 2. $3_{3}$ & 1. $4_{3}$ & 1. $6_{4}$ & 0. $9_{7}$ & 1. $3_{7}$ & 1. $4_{7}$ & 1. $2_{7}$ \\
\hline
\end{tabular}

- Plus sign indicates gain in weight.

- The low expansivity of Vycor glass and its high softening point suggested that it might be suitable as a container for pyrosulfate fusions. Fifty grams of potassium pyrosulfate was kept fused for 6 hours in each of 3 Vycor flasks by heating them over gas burners. The temperature was not measured or closely controlled. The area exposed in each flask was about $50 \mathrm{~cm} .^{2}$ The losses in weight ranged from 2 to $5 \mathrm{mg}$. 
TABLE 4.-Observed losses $\mathrm{\text {in }}$ weight (milligrams) of flasks exposed to acid reagents and water-Continued

\begin{tabular}{|c|c|c|c|c|c|c|c|c|c|c|c|c|c|c|c|}
\hline \multicolumn{4}{|c|}{ Glasbake, periods } & \multicolumn{4}{|c|}{ Kimble, periods } & \multicolumn{4}{|c|}{ Pyrex, periods } & \multicolumn{4}{|c|}{ Vycor, periods } \\
\hline 1 & 2 & 3 & $\begin{array}{l}\text { Aver- } \\
\text { age }\end{array}$ & 1 & 2 & 3 & $\begin{array}{l}\text { Aver- } \\
\text { age }\end{array}$ & 1 & 2 & 3 & $\begin{array}{l}\text { Aver- } \\
\text { age }\end{array}$ & 1 & & 3 & $\begin{array}{l}\text { Aver- } \\
\text { age }\end{array}$ \\
\hline
\end{tabular}

7. PERCHLORIC ACID

\begin{tabular}{|c|c|c|c|c|c|c|c|c|c|c|c|c|c|c|c|}
\hline $\begin{array}{r}0.5 \\
.1 \\
1.3\end{array}$ & $\begin{array}{r}+0.1 \\
+.1 \\
.0\end{array}$ & 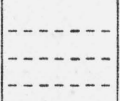 & $\begin{array}{r}0.2_{0} \\
.0_{0} \\
.6_{5}\end{array}$ & $\begin{array}{r}0.1 \\
.0 \\
.2\end{array}$ & $\begin{array}{r}0.0 \\
.0 \\
+.2\end{array}$ & 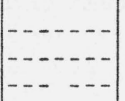 & $\begin{array}{r}0.0_{5} \\
.00 \\
.00\end{array}$ & $\begin{array}{r}0.3 \\
.3 \\
.3\end{array}$ & $\begin{array}{r}+0.3 \\
+.1 \\
+.1\end{array}$ & 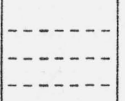 & $\begin{array}{r}0.0_{0} \\
.1_{0} \\
.1_{0}\end{array}$ & $\left|\begin{array}{c}------ \\
---\cdots-- \\
------\end{array}\right|$ & $\mid \begin{array}{l}------ \\
----- \\
-\cdots---\end{array}$ & $\mid \begin{array}{l}----- \\
---\cdots- \\
---\cdots--\end{array}$ & 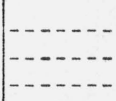 \\
\hline Avg $\ldots . .0 .63$ & $+0.0_{7}$ & $\ldots \ldots$ & 0. $2_{8}$ & $0.1_{0}$ & $+0.0_{7}$ & $\ldots$ & 0. $\mathrm{O}_{2}$ & $0.3_{0}$ & +0.17 & $\mid \ldots \ldots$ & 0. $0_{7}$ & & & $\ldots$ & \\
\hline
\end{tabular}

s Plus sign indicates gain in weight. 


\section{ALKALINE REAGENTS}

The numbers of the following explanatory notes correspond to the numbers attached to each group of data in table 5 and figure 2:

8. Twentieth-normal sodium hydroxide.

9. Half-normal sodium hydroxide.

10. Half-normal potassium hydroxide.

11. Half-normal sodium carbonate.

12. Half-normal potassium hydroxide in 95-percent alcohol. When this solution was used the flasks were connected with water-cooled

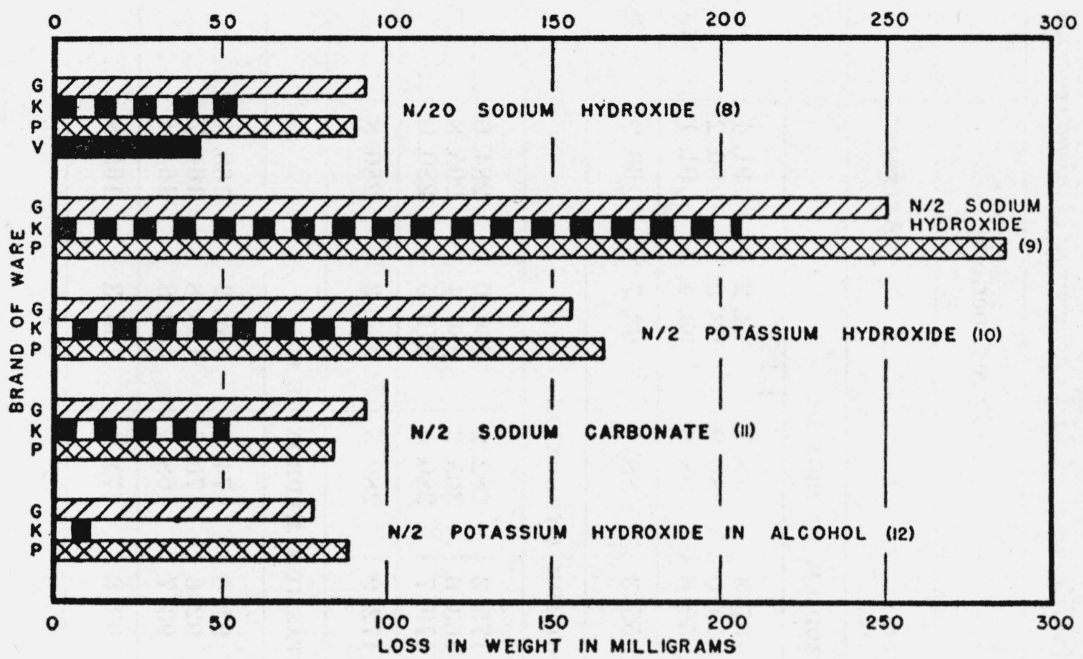

FIGURE 2.-Comparative resistance to alkaline reagents.

A verage loss in weight, in milligrams, per flask per 6-hour period of exposure.

For $0.5 \mathrm{~N}$ potassium hydroxide in alcohol the loss in weight shown is six times the average hourly loss reported in table 5.

reflux condensers, by means of rubber stoppers. The first period was 1 hour and the second 2 hours, instead of the usual two 6-hour periods. The object of this departure from the usual procedure was to simulate actual conditions under which alcoholic potash solutions are frequently used.

13. A solution containing, at the outset, $100 \mathrm{ml}$ of ammonium hydroxide (about 28 percent of $\mathrm{NH}_{3}$ ) and $100 \mathrm{~g}$ of ammonium chloride in 1 liter. This solution was boiled in the usual way and was replenished with water. These results are not shown in figure 2 .

14. Ammonium hydroxide, about 28 percent of $\mathrm{NH}_{3}$. This reagent was used at room temperature. The flasks were closed with rubber stoppers. The first period was 8 days and the second 34 days. These results are not shown in figure 2 . 
TABLE 5.-Observed losses in weight (milligrams) of flasks exposed to alkaline reagents

\begin{tabular}{l|l|l|l|l|l|l|l|l|}
\multicolumn{3}{l|}{ Glasbake, periods } & \multicolumn{3}{c|}{ Kimble, periods } & \multicolumn{3}{c}{ Pyrex, periods } \\
\hline 1 & 2 & Average & 1 & 2 & Average & 1 & 2 & Average \\
\hline
\end{tabular}

8. $0.05 N$ SODIUM HYDROXIDE

\begin{tabular}{|c|c|c|c|c|c|c|c|c|c|c|c|c|}
\hline & $\begin{array}{l}90.4 \\
91.8 \\
91.5\end{array}$ & $\begin{array}{l}95.6 \\
98.9 \\
96.6\end{array}$ & $\begin{array}{l}93.0 \\
95.4 \\
94.0\end{array}$ & $\begin{array}{l}55.2 \\
53.5 \\
55.4\end{array}$ & $\begin{array}{l}59.4 \\
52.5 \\
55.7\end{array}$ & $\begin{array}{l}57.3 \\
53.0 \\
55.6\end{array}$ & $\begin{array}{l}88.7 \\
86.9 \\
88.8\end{array}$ & $\begin{array}{l}94.2 \\
93.5 \\
93.4\end{array}$ & $\begin{array}{l}91.4 \\
90.2 \\
91.1\end{array}$ & $\begin{array}{l}41.0 \\
39.1 \\
36.5\end{array}$ & $\begin{array}{l}49.6 \\
46.9 \\
46.9\end{array}$ & $\begin{array}{l}45.3 \\
43.0 \\
41.7\end{array}$ \\
\hline Avg... & 91.2 & 97.0 & 94.1 & 54. 7 & 55.9 & 55.3 & 88.1 & 93.7 & 90.9 & 38.9 & 47. 8 & 43. 3 \\
\hline
\end{tabular}

9. $0.5 N$ SODIUM HYDROXIDE

\begin{tabular}{|c|c|c|c|c|c|c|c|c|c|c|c|c|}
\hline & $\begin{array}{l}247.9 \\
241.2 \\
257.0\end{array}$ & $\begin{array}{l}257.6 \\
251.0 \\
252.6\end{array}$ & $\begin{array}{l}252.8 \\
246.1 \\
254.8\end{array}$ & $\begin{array}{l}172.7 \\
169.5 \\
176.1\end{array}$ & $\begin{array}{l}177.9 \\
157.8 \\
183.3\end{array}$ & $\begin{array}{l}175.3 \\
163.6 \\
179.7\end{array}$ & $\begin{array}{l}282.2 \\
305.3 \\
280.5\end{array}$ & $\begin{array}{l}286.9 \\
286.4 \\
279.5\end{array}$ & $\begin{array}{l}284.6 \\
295.8 \\
280.0\end{array}$ & $\begin{array}{l}-- \\
-- \\
--\end{array}$ & 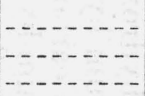 & 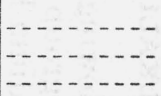 \\
\hline Avg _- & 248. 7 & 253. 7 & 251. 2 & 172. 8 & 173.0 & 172.9 & 289. 3 & 284. 3 & 286. 8 & - & -- & $\ldots$ \\
\hline
\end{tabular}

10. 0.5 N POTASSIUM HYDROXIDE *

\begin{tabular}{|c|c|c|c|c|c|c|c|c|c|c|c|c|}
\hline & $\begin{array}{l}161.1 \\
158.9 \\
159.7 \\
\end{array}$ & $\begin{array}{l}153.6 \\
149.7 \\
152.5\end{array}$ & $\begin{array}{l}157.4 \\
154.3 \\
156.1\end{array}$ & $\begin{array}{l}94.3 \\
92.5 \\
93.8\end{array}$ & $\begin{array}{l}94.9 \\
92.6 \\
93.6 \\
\end{array}$ & $\begin{array}{l}94.6 \\
92.6 \\
93.7 \\
\end{array}$ & $\begin{array}{l}174.7 \\
170.6 \\
168.9 \\
\end{array}$ & $\begin{array}{l}162.2 \\
158.8 \\
159.8\end{array}$ & $\begin{array}{l}168.4 \\
164.7 \\
164.4\end{array}$ & & 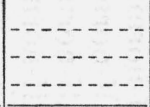 & 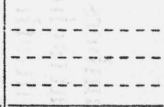 \\
\hline Avg - & 159. 9 & 151. 9 & 155. 9 & 93. 5 & 93.7 & 93.6 & 171. 4 & 160. 3 & 165. 8 & & $\ldots$ & $\ldots$ \\
\hline
\end{tabular}


11. 0.5 N SODIUM CARBONATE

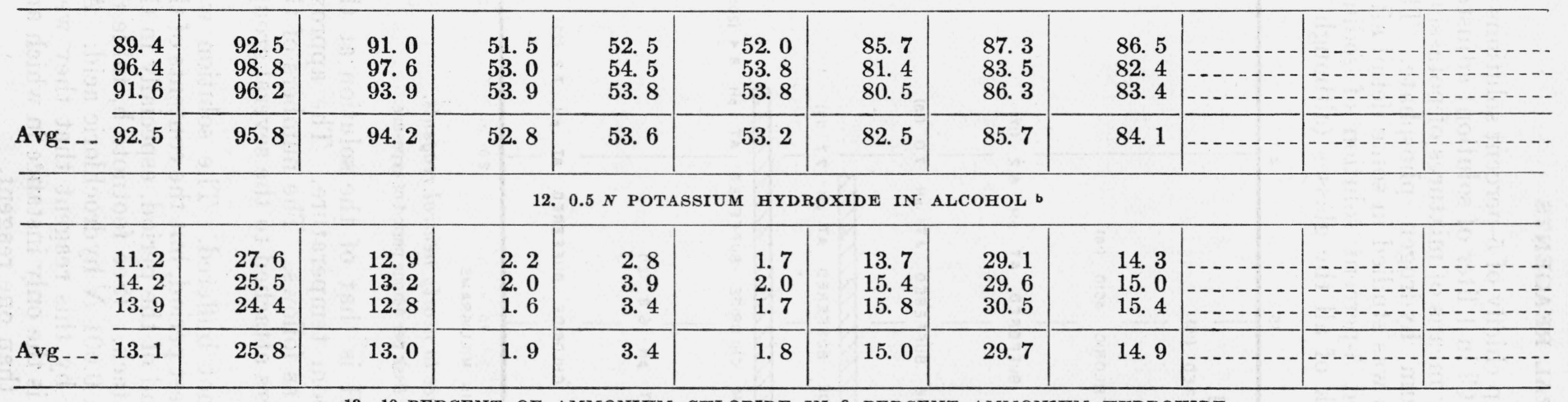

13. 10 PERCENT OF AMMONIUM CHLORIDE IN 3 PERCENT AMMONIUM HYDROXIDE

\begin{tabular}{|c|c|c|c|c|c|c|c|c|c|c|c|c|}
\hline & $\begin{array}{l}\text { 1. } 9 \\
\text { 2. } 6 \\
\text { 2. } 8\end{array}$ & $\begin{array}{l}2.2 \\
1.7 \\
1.5\end{array}$ & $\begin{array}{l}2.0 \\
2.2 \\
2.2\end{array}$ & $\begin{array}{l}0.9 \\
0.7 \\
1.1\end{array}$ & $\begin{array}{l}\text { 1. } 5 \\
0.9 \\
\text { 1. } 4\end{array}$ & $\begin{array}{l}\text { 1. } 2 \\
0.8 \\
\text { 1. } 2\end{array}$ & $\begin{array}{l}\text { 1. } 2 \\
\text { 1. } 3 \\
\text { 1. } 9\end{array}$ & $\begin{array}{l}\text { 1. } 4 \\
\text { 1. } 4 \\
0.9\end{array}$ & $\begin{array}{l}\text { 1. } 3 \\
\text { 1. } 4 \\
\text { 1. } 4\end{array}$ & & 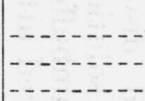 & (-- \\
\hline Avg $\ldots$ & 2. 4 & 1. 8 & 2. 1 & 0. 9 & 1. 3 & 1. 1 & 1. 5 & 1. 2 & 1. 4 & & & -. \\
\hline
\end{tabular}

14. CONCENTRATED AMMONIUM HYDROXIDE AT ROOM TEMPERATURE •

\begin{tabular}{|c|c|c|c|c|c|c|c|c|c|c|c|c|}
\hline & $\begin{array}{l}\text { 2. } 1 \\
\text { 1. } 3 \\
\text { 1. } 2\end{array}$ & $\begin{array}{l}\text { 1. } 5 \\
\text { 1. } 7 \\
\text { 1. } 8\end{array}$ & 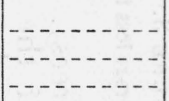 & $\begin{array}{l}\text { 1. } 2 \\
\text { 1. } 0 \\
0.9\end{array}$ & $\begin{array}{l}1.0 \\
0.9 \\
0.7\end{array}$ & & $\begin{array}{l}\text { 1. } 2 \\
0.3 \\
0.4\end{array}$ & $\begin{array}{l}\text { 1. } 1 \\
\text { 1. } 3 \\
\text { 1. } 4\end{array}$ & 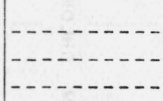 & & 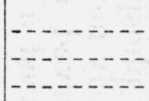 & 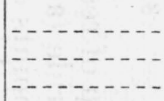 \\
\hline Avg $\ldots 1$ & 1. 5 & 1. 7 & $\ldots \ldots$ & 1. 0 & 0. 9 & 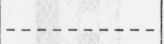 & 0.6 & 1. 3 & $\ldots$ & $\ldots$ & -. & - \\
\hline
\end{tabular}

- Arsenic was determined (by J.A. Scherrer) in the potassium hydroxide solution taken from 2 of each of the 3 brands of flasks after the first 6 -hour period of attack. The results, in milligrams of arsenic removed from each flask, are as follows: Glasbake - $0.14,0.16$; Kimble- $0.06,0.07$; Pyrex - not detected (less than 0.004 mg).

b The first period was for 1 hour; the second period was for 2 hours. The average per period and the grand average are per hour.

- The first period was for 8 days; the second period was for 34 days. 


\section{NEARLY NEUTRAL REAGENTS}

This group of reagents was made up chiefly of 5-percent solutions of sodium chloride (actually $50 \mathrm{~g}$ of $\mathrm{NaCl}$ in 1 liter of solution) adjusted to selected points on the $\mathrm{pH}$ scale by means of mixtures of potassium dihydrogen phosphate and disodium hydrogen phosphate. The effect of nearly neutral salt solutions was studied in some detail after it was observed that an unbuffered 5-percent solution of sodium chloride caused a pronounced attack of all the glasses (although in

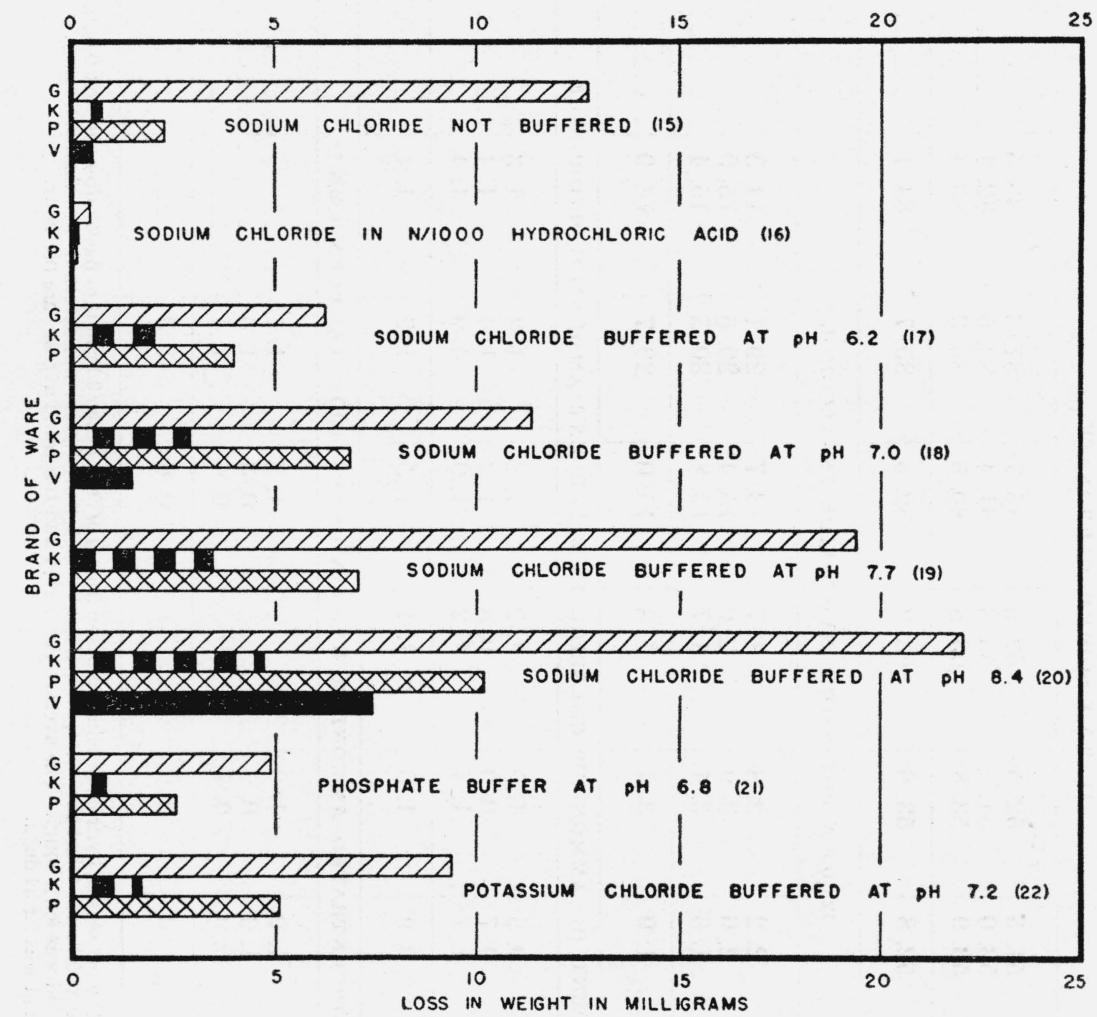

FiguRE 3.-Comparative resistance to nearly neutral reagents.

A verage loss in weight, in milligrams, per flask per 6 -hour period of exposure.

varying degrees). The indicated $\mathrm{pH}$ is that of the solution at the beginning of the test period, at room temperature. The approximately neutral solutions used were as follows. The numbers of the paragraphs correspond to the numbers attached to the several groups of data in table 6 and figure 3.

15. Sodium chloride, 5-percent, not buffered. The solution was slightly acid at the beginning of the test period, but the contents of the flasks were slightly alkaline at the end of the period, especially in the group which showed the greatest attack. (See footnote b, table 6.)

16. Sodium chloride, 5-percent, in $0.001 N$ hydrochloric acid. All the flasks were so slightly attacked by this reagent that they were used again with the next one. This is the only instance in which any flasks were used for tests with more than one reagent. 
17. Sodium chloride, 5-percent, buffered at $\mathrm{pH} 6.2$. This solution contained, in addition to the sodium chloride, $10.85 \mathrm{~g}$ of $\mathrm{Na}_{2} \mathrm{HPO}_{4}$. $12 \mathrm{H}_{2} \mathrm{O}$ and $4.55 \mathrm{~g}$ of $\mathrm{KH}_{2} \mathrm{PO}_{4}$, in 1 liter.

18. Sodium chloride, 5-percent, buffered at $\mathrm{pH}$ 7.0. In addition to the sodium chloride, the solution contained $19.0 \mathrm{~g}$ of $\mathrm{Na}_{2} \mathrm{HPO}_{4}$. $12 \mathrm{H}_{2} \mathrm{O}$ and $1.15 \mathrm{~g}$ of $\mathrm{KH}_{2} \mathrm{PO}_{4}$, in 1 liter.

19. Sodium chloride, 5-percent, buffered at $\mathrm{pH}$ 7.7. The buffersalt concentrations were $33 \mathrm{~g}$ of $\mathrm{Na}_{2} \mathrm{HPO}_{4} \cdot 12 \mathrm{H}_{2} \mathrm{O}$ and $0.25 \mathrm{~g}$ of $\mathrm{KH}_{2} \mathrm{PO}_{4}$, in 1 liter.

20. Sodium chloride, 5-percent, buffered at $\mathrm{pH}$ 8.4. The buffer salt concentration was $33 \mathrm{~g}$ of $\mathrm{Na}_{2} \mathrm{HPO}_{4} \cdot 12 \mathrm{H}_{2} \mathrm{O}$, in 1 liter.

21. Buffer solution, at $\mathrm{pH} 6.8$, without sodium chloride. The solution contained $10.85 \mathrm{~g}$ of $\mathrm{Na}_{2} \mathrm{HPO}_{4} .12 \mathrm{H}_{2} \mathrm{O}$ and $4.55 \mathrm{~g}$ of $\mathrm{KH}_{2} \mathrm{PO}_{4}$, in 1 liter. The results may be compared with those of Nos. 17 and 18 to show the marked effect of sodium chloride.

22. Potassium chloride, 7-percent (70 $\mathrm{g}$ in 1 liter), buffered at $\mathrm{pH}$ 7.2. The buffer salt concentrations were $18.5 \mathrm{~g}$ of $\mathrm{Na}_{2} \mathrm{HPO}_{4}$. $12 \mathrm{H}_{2} \mathrm{O}$ and $1.15 \mathrm{~g}$ of $\mathrm{KH}_{2} \mathrm{PO}_{4}$, in 1 liter. This solution contained approximately the same molar concentrations of salts as No. 18 and was at nearly the same $\mathrm{pH}$. The two sets of results show a marked difference between the effect of sodium and potassium chloride on the three glasses. 


\begin{tabular}{|c|c|c|c|c|c|c|c|c|c|c|c|c|c|c|c|}
\hline \multicolumn{4}{|c|}{ Glasbake, periods } & \multicolumn{4}{|c|}{ Kimble, periods } & \multicolumn{4}{|c|}{ Pyrex, periods } & \multicolumn{4}{|c|}{ Vycor, periods } \\
\hline 1 & 2 & 3 & $\begin{array}{c}\text { Aver- } \\
\text { age }\end{array}$ & 1 & 2 & 3 & $\begin{array}{l}\text { Aver- } \\
\text { age }\end{array}$ & 1 & 2 & 3 & $\begin{array}{l}\text { Aver- } \\
\text { age }\end{array}$ & 1 & 2 & 3 & $\begin{array}{c}\text { Aver- } \\
\text { age }\end{array}$ \\
\hline
\end{tabular}

15. 5 PERCENT SODIUM CHLORIDE SOLUTION, NOT BUFFERED b

\begin{tabular}{|c|c|c|c|c|c|c|c|c|c|c|c|c|c|c|c|}
\hline $\begin{array}{l}16.6 \\
10.6 \\
15.8\end{array}$ & $\begin{array}{r}13.0 \\
6.5 \\
12.1\end{array}$ & $\begin{array}{r}\text { 15. } 7 \\
7.9 \\
16.9\end{array}$ & $\begin{array}{r}\text { 15. } 1_{0} \\
\text { 8. } 3_{3} \\
\text { 14. } 9_{3}\end{array}$ & $\begin{array}{r}0.6 \\
.7 \\
.7\end{array}$ & $\begin{array}{r}0.8 \\
.3 \\
.6\end{array}$ & $\begin{array}{l}0.7 \\
1.0 \\
0.6\end{array}$ & $\begin{array}{r}0.7_{0} \\
.6_{7} \\
.6_{3}\end{array}$ & $\begin{array}{l}\text { 4. } 4 \\
\text { 3. } 8 \\
\text { 3. } 4\end{array}$ & $\begin{array}{l}1.2 \\
\text { 1. } 2 \\
\text { 1. } 4\end{array}$ & $\begin{array}{l}\text { 1. } 4 \\
\text { 1. } 6 \\
\text { 1. } 7\end{array}$ & $\begin{array}{l}\text { 2. } 3_{3} \\
\text { 2. } 2_{0} \\
\text { 2. } 1_{7}\end{array}$ & $\begin{array}{r}0.2 \\
.0 \\
.2\end{array}$ & $\begin{array}{l}1.2 \\
1.0 \\
1.4\end{array}$ & $\begin{array}{r}0.0 \\
+.1 \\
.4\end{array}$ & $\begin{array}{r}0.4_{7} \\
.3_{0} \\
.67\end{array}$ \\
\hline Avg_- $14.3_{3}$ & 10. $5_{3}$ & 13. $5_{0}$ & 12. $7_{9}$ & $0.6_{7}$ & $0.5_{7}$ & $0.7_{7}$ & $0.6_{7}$ & 3. $8_{7}$ & 1. $2_{7}$ & 1. $5_{7}$ & 2. $2_{3}$ & $0.1_{3}$ & 1. $2_{0}$ & $0.1_{0}$ & $0.4_{8}$ \\
\hline
\end{tabular}

16. 5 PERCENT OF SODIUM CHLORIDE IN $0.001 N$ HYDROCHLORIC ACID

\begin{tabular}{|c|c|c|c|c|c|c|c|c|c|c|c|c|c|c|c|}
\hline $\begin{array}{r}0.4 \\
.7 \\
.6\end{array}$ & $\begin{array}{r}0.2 \\
.1 \\
.4\end{array}$ & 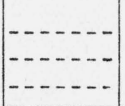 & $\begin{array}{r}0.3_{0} \\
.4_{0} \\
.5_{0}\end{array}$ & $\begin{array}{r}0.0 \\
.1 \\
+.1\end{array}$ & $\begin{array}{r}0.1 \\
.0 \\
.1\end{array}$ & 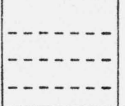 & $\begin{array}{r}0.0_{5} \\
.0_{5} \\
.0_{0}\end{array}$ & $\begin{array}{r}0.2 \\
.1 \\
.0\end{array}$ & $\begin{array}{r}0.2 \\
.0 \\
.1\end{array}$ & 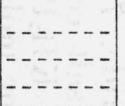 & $\begin{array}{r}0.2_{0} \\
.0_{5} \\
.0_{5}\end{array}$ & 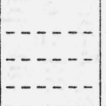 & 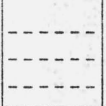 & 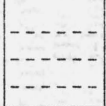 & 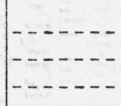 \\
\hline Avg__. $0.5_{7}$ & $0.2_{3}$ & $\ldots \ldots$ & $0.4_{0}$ & $0.0_{0}$ & $0.0_{7}$ & $-\ldots-$. & $0.0_{3}$ & $0.1_{0}$ & $0.1_{0}$ & $\ldots$ & $0.1_{0}$ & - & -- & & $\ldots$ \\
\hline
\end{tabular}

17. 5 PERCENT SODIUM CHLORIDE SOLUTION BUFFERED AT pH 6.2

\begin{tabular}{|c|c|c|c|c|c|c|c|c|c|c|c|c|c|c|c|}
\hline $\begin{array}{l}\text { 4. } 6 \\
5.7 \\
4.9\end{array}$ & $\begin{array}{l}\text { 7. } 3 \\
\text { 8. } 4 \\
\text { 7. } 1\end{array}$ & 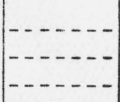 & $\begin{array}{l}\text { 5. } 9_{5} \\
\text { 7. } 0_{5} \\
\text { 6. } 0_{0}\end{array}$ & $\begin{array}{l}\text { 2. } 1 \\
\text { 2. } 1 \\
\text { 2. } 1\end{array}$ & $\begin{array}{l}1.8 \\
1.7 \\
1.9\end{array}$ & 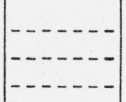 & $\begin{array}{l}\text { 1. } 9_{5} \\
\text { 1. } 9_{0} \\
\text { 2. } 0_{0}\end{array}$ & $\begin{array}{l}4.0 \\
4.5 \\
4.0\end{array}$ & $\begin{array}{l}\text { 4. } 2 \\
\text { 3. } 8 \\
\text { 3. } 6\end{array}$ & 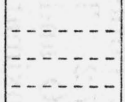 & $\begin{array}{l}\text { 4. } 1_{0} \\
\text { 4. } 1_{5} \\
\text { 3. } 8_{0}\end{array}$ & $\mid \begin{array}{c}-\cdots-- \\
-\cdots--- \\
-\cdots---\end{array}$ & $\mid \begin{array}{l}--- \\
---\cdots \\
-\cdots-\cdots\end{array}$ & $\mid \begin{array}{r}---\cdots \\
-\cdots-- \\
---\cdots-\end{array}$ & $\mid \begin{array}{l}-\ldots \\
-\cdots\end{array}$ \\
\hline Avg $\ldots . .5 .0_{7}$ & 7. $6_{0}$ & $\ldots \ldots$ & 6. $3_{3}$ & 2. $1_{0}$ & 1. $8_{0}$ & $\ldots \ldots$ & 1. $9_{5}$ & 4. $1_{7}$ & 3. $8_{7}$ & 1 & 4. $\mathrm{O}_{2}$ & & & -- & $\ldots$ \\
\hline
\end{tabular}


18. 5 PERCENT SODIUM CHLORIDE SOLUTION BUFFERED AT pH 7.0

\begin{tabular}{|c|c|c|c|c|c|c|c|c|c|c|c|c|c|c|c|}
\hline $\begin{array}{l}12.5 \\
10.6 \\
11.4\end{array}$ & $\begin{array}{r}13.3 \\
9.6 \\
11.4\end{array}$ & 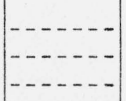 & $\begin{array}{l}\text { 12. } 9_{0} \\
10.1_{0} \\
11.4_{0}\end{array}$ & $\begin{array}{l}2.5 \\
2.7 \\
2.5\end{array}$ & $\begin{array}{l}3.4 \\
3.3 \\
\text { 2. } 8\end{array}$ & 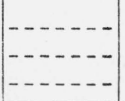 & $\begin{array}{l}\text { 2. } 9_{5} \\
\text { 3. } 0_{0} \\
\text { 2. } 6_{5}\end{array}$ & $\begin{array}{l}\text { 7. } 0 \\
8.5 \\
\text { 7. } 4\end{array}$ & $\begin{array}{l}6.9 \\
6.1 \\
5.5\end{array}$ & 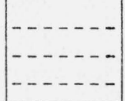 & $\begin{array}{l}\text { 6. } 9_{5} \\
\text { 7. } 3_{0} \\
\text { 6. } 4_{5}\end{array}$ & $\begin{array}{l}1.1 \\
1.5 \\
1.2\end{array}$ & $\begin{array}{l}1.5 \\
1.5 \\
1.6\end{array}$ & $\left|\begin{array}{c}- \\
-\cdots-.-- \\
-\cdots--- \\
------\end{array}\right|$ & $\begin{array}{l}\text { 1. } 3_{0} \\
\text { 1. } 5_{0} \\
\text { 1. } 4_{0}\end{array}$ \\
\hline Avg $--11.5_{0}$ & 11. $4_{3}$ & $\ldots \ldots$ & 11. $4_{7}$ & 2. $5_{7}$ & 3. $1_{7}$ & $\ldots \ldots$ & 2. $8_{7}$ & 7. $6_{3}$ & 6. $1_{7}$ & $\ldots \ldots$ & 6. $9_{0}$ & 1. $2_{7}$ & 1. $5_{3}$ & $\ldots$ & 1. $4_{0}$ \\
\hline
\end{tabular}

19. 5 PERCENT SODIUM CHLORIDE SOLUTION BUFFERED AT pH 7.7

\begin{tabular}{|c|c|c|c|c|c|c|c|c|c|c|c|c|c|c|c|}
\hline $\begin{array}{l}15.9 \\
19.2 \\
16.0\end{array}$ & $\begin{array}{l}17.2 \\
25.2 \\
22.8\end{array}$ & 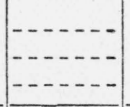 & $\begin{array}{l}16.5_{5} \\
22.2_{0} \\
19.4_{0}\end{array}$ & $\begin{array}{l}2.5 \\
2.7 \\
2.8\end{array}$ & $\begin{array}{l}4.4 \\
3.4 \\
3.6\end{array}$ & 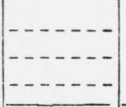 & $\begin{array}{l}\text { 3. } 4_{5} \\
\text { 3. } 0_{5} \\
\text { 3. } 2_{0}\end{array}$ & $\begin{array}{l}\text { 6. } 8 \\
\text { 6. } 6 \\
\text { 7. } 1 \\
\end{array}$ & $\begin{array}{l}\text { 7. } 2 \\
\text { 7. } 3 \\
\text { 7. } 4 \\
\end{array}$ & 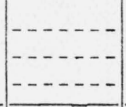 & $\begin{array}{l}\text { 7. } 0_{0} \\
\text { 6. } 9_{5} \\
\text { 7. } 2_{5} \\
\end{array}$ & 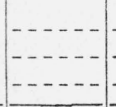 & 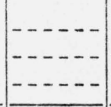 & $\begin{array}{l}-\cdots- \\
-\cdots- \\
-\cdots-\end{array}$ & 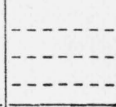 \\
\hline Avg $\ldots 17.0_{3}$ & 21. $7_{3}$ & $\ldots \ldots$ & 19. $3_{8}$ & 2. $6_{7}$ & 3. $8_{0}$ & $\ldots$ & 3. $2_{3}$ & 6. $8_{3}$ & 7. $3_{0}$ & - & 7. $0_{7}$ & -- & & -- & -- \\
\hline
\end{tabular}

20. 5 PERCENT SODIUM CHLORIDE SOLUTION BUFFERED AT pH 8.4

\begin{tabular}{|c|c|c|c|c|c|c|c|c|c|c|c|c|c|c|c|}
\hline $\begin{array}{l}23.7 \\
24.2 \\
20.1\end{array}$ & $\begin{array}{l}21.4 \\
25.3 \\
17.8\end{array}$ & 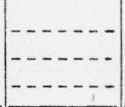 & $\begin{array}{l}\text { 22. } 5_{5} \\
\text { 24. } 7_{5} \\
\text { 18. } 9_{5}\end{array}$ & $\begin{array}{l}4.5 \\
5.0 \\
5.0\end{array}$ & $\begin{array}{l}4.5 \\
4.1 \\
5.0\end{array}$ & 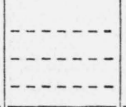 & $\begin{array}{l}\text { 4. } 5_{0} \\
\text { 4. } 5_{5} \\
\text { 5. } 0_{0}\end{array}$ & $\begin{array}{l}\text { 11. } 3 \\
12.2 \\
11.3\end{array}$ & $\begin{array}{l}9.2 \\
8.9 \\
8.1\end{array}$ & 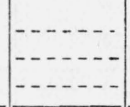 & $\begin{array}{r}10.2_{5} \\
10.5_{5} \\
9.7_{0}\end{array}$ & $\begin{array}{l}\text { 6. } 2 \\
\text { 7. } 2 \\
\text { 7. } 6\end{array}$ & $\begin{array}{l}\text { 7. } 6 \\
8.1 \\
\text { 7. } 6\end{array}$ & $\mid \begin{array}{c}---\cdot-- \\
-\cdots--- \\
-\cdots---\end{array}$ & $\begin{array}{l}\text { 6. } 9_{0} \\
\text { 7. } 6_{5} \\
\text { 7. } 6_{0}\end{array}$ \\
\hline Avg $\ldots . .22 .67$ & $21.5_{0}$ & $\ldots \ldots$ & 22. $0_{8}$ & 4. $8_{3}$ & 4. $5_{3}$ & & 4. $6_{8}$ & 11. $6_{0}$ & 8. $7_{3}$ & - & 10. $1_{7}$ & 7. $0_{0}$ & 7. 77 & & 7. $3_{8}$ \\
\hline
\end{tabular}

21. BUFFER SOLUTION AT pH 6.8

\begin{tabular}{|c|c|c|c|c|c|c|c|c|c|c|c|c|c|c|c|}
\hline $\begin{array}{l}5.4 \\
4.5 \\
4.5\end{array}$ & $\begin{array}{l}5.5 \\
4.8 \\
4.7\end{array}$ & 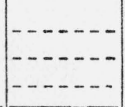 & $\begin{array}{l}\text { 5. } 4_{5} \\
\text { 4. } 6_{5} \\
\text { 4. } 6_{0}\end{array}$ & $\begin{array}{r}0.8 \\
.5 \\
.7\end{array}$ & $\begin{array}{r}0.9 \\
\text { 1. } 0 \\
.7\end{array}$ & 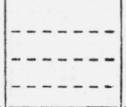 & $\begin{array}{r}0.8_{5} \\
.7_{5} \\
.7_{0}\end{array}$ & $\begin{array}{l}2.3 \\
2.8 \\
2.9\end{array}$ & $\begin{array}{l}2.6 \\
2.4 \\
2.5\end{array}$ & 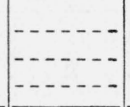 & $\begin{array}{l}\text { 2. } 4_{5} \\
\text { 2. } 6_{0} \\
\text { 2. } 7_{0}\end{array}$ & 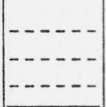 & 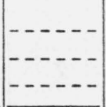 & 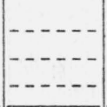 & $\mid$ \\
\hline Avg_... $4.8_{0}$ & 5. $0_{0}$ & $-\ldots . .-$ & 4. $9_{0}$ & $0.6_{7}$ & $0.8_{7}$ & & $0.7_{7}$ & 2. $6_{7}$ & 2. $5_{0}$ & -- & 2. $5_{8}$ & - & - & & -- \\
\hline
\end{tabular}

a Plus sign indicates gain in weight.

b This solution was slightly acid (acid to methyl red) at the beginning of the treatment. At the end of the 6-hour period the solutions in the Kimble and Pyrex flasks were alkalin to methyl red but acid to phenolphthalein. That in the Glasbake flasks was alkaline to phenolphthalein. 
TABLE 6.-Observed losses in werght (milligrams) of flasks exposed to nearly neutral reagents-Continued

\begin{tabular}{|c|c|c|c|c|c|c|c|c|c|c|c|c|c|c|c|}
\hline \multicolumn{4}{|c|}{ Glasbake, periods } & \multicolumn{4}{|c|}{ Kimble, periods } & \multicolumn{4}{|c|}{ Pyrex, periods } & \multicolumn{4}{|c|}{ Vycor, periods } \\
\hline 1 & 2 & 3 & $\begin{array}{l}\text { Aver- } \\
\text { age }\end{array}$ & 1 & 2 & 3 & $\begin{array}{c}\text { Aver- } \\
\text { age }\end{array}$ & 1 & 2 & 3 & $\begin{array}{c}\text { Aver- } \\
\text { age }\end{array}$ & 1 & 2 & & $\begin{array}{l}\text { Aver- } \\
\text { age }\end{array}$ \\
\hline
\end{tabular}

22. 7 PERCENT POTASSIUM CHLORIDE SOLUTION BUFFERED AT pH $\mathbf{7 . 2}$

\begin{tabular}{|c|c|c|c|c|c|c|c|c|c|c|c|c|c|c|c|}
\hline Avg _... $9.8_{0}$ & 8. $9_{7}$ & $\ldots$ & 9. 38 & 1. $6_{0}$ & 1. $7_{7}$ & $\ldots$ & 1. $6_{8}$ & 5. $6_{3}$ & 4. $5_{7}$ & $\ldots \ldots$ & 5. $1_{0}$ & & & & \\
\hline
\end{tabular}




\section{RESISTANCE TO MECHANICAL SHOCK}

The resistance of the 250-ml flasks to mechanical shock was determined ${ }^{9}$ by the method currently used for determining the resistance of chinaware to chipping. Briefly, this consisted in placing a flask firmly between two cast-iron blocks forming a $90^{\circ} \mathrm{V}$, and striking the flask at its maximum diameter with the tup of a pendulum of fixed length, swinging through ares of increasing length until the glass broke. ${ }^{10}$ After each impact, the flask was rotated slightly so that successive blows were delivered at different points on the glass. The energy (in ft-lb) required to break the glass was computed from the weight of the tup and the distance through which it swung. Vycor flasks were not included in these tests.

The results obtained on 12 flasks of each kind are given in table 7, with the flasks arranged in order of increasing weight. The average results indicate significant differences in resistance to mechanical shock. In this respect the three wares fall in the order of their relative weights. However, there is a wide range in the individual values in each group, probably because of variations in thickness and in the amount of surface checking and scratching incident to handling.

TABLE 7.-Resistance to mechanical shock

\begin{tabular}{|c|c|c|c|c|c|}
\hline \multicolumn{2}{|c|}{ Glasbake } & \multicolumn{2}{|c|}{ Kimble } & \multicolumn{2}{|c|}{ Pyrex } \\
\hline Weight & $\begin{array}{l}\text { Impact } \\
\text { energy }\end{array}$ & Weight & $\begin{array}{l}\text { Impact } \\
\text { energy }\end{array}$ & Weight & $\begin{array}{l}\text { Impact } \\
\text { energy }\end{array}$ \\
\hline $\begin{array}{r}g \\
79.3 \\
81.8 \\
88.1 \\
89.9 \\
90.5 \\
91.2 \\
91.4 \\
94.0 \\
94.1 \\
95.2 \\
95.3 \\
96.5\end{array}$ & $\begin{array}{r}f t-l b \\
0.20 \\
.30 \\
.35 \\
.15 \\
.50 \\
.55 \\
.65 \\
.35 \\
.50 \\
.40 \\
.60 \\
.40\end{array}$ & $\begin{array}{c}g \\
57.1 \\
59.2 \\
59.9 \\
60.8 \\
61.3 \\
62.5 \\
62.6 \\
63.1 \\
63.1 \\
63.6 \\
66.3 \\
66.7\end{array}$ & $\begin{array}{c}f t-l b \\
0.20 \\
.10 \\
.15 \\
.14 \\
.17 \\
.15 \\
.15 \\
.10 \\
.22 \\
.13 \\
.15 \\
.14\end{array}$ & $\begin{array}{c}g \\
74.0 \\
74.4 \\
76.2 \\
78.9 \\
79.1 \\
80.0 \\
81.0 \\
82.2 \\
83.0 \\
\text { 83. } 2 \\
\text { 83. } 3 \\
85.0\end{array}$ & $\begin{array}{r}f t-l b \\
0.25 \\
.25 \\
.21 \\
.23 \\
.20 \\
.20 \\
.20 \\
.30 \\
.32 \\
.45 \\
.45 \\
.45\end{array}$ \\
\hline Avg _- 90.6 & .44 & 62.2 & .15 & 80.0 & .29 \\
\hline
\end{tabular}

s Abnormal result. The break occurred at 1 of the points of support, rather than at the point of impact. This result was rejected in computing the average.

\section{RESISTANCE TO THERMAL SHOCK}

Resistance to thermal shock was determined ${ }^{11}$ by slowly heating a flask, containing $100 \mathrm{ml}$ of a high-flash mineral oil, on a hot plate to a selected temperature, and then quickly immersing it to the neck in

\footnotetext{
- These tests were made by Donald Hubbard.

$10 \mathrm{~A}$ complete description of the apparatus used and its method of application is given in section $\mathrm{F}-3 \mathrm{a}$ and fig. 9 of Federal Specification M-C-301 for "Chinaware; Vitrified." (Superintendent of Documents, Washington, D. C., price, 10 cents.)

11 These tests were made by Donald Hubbard.
} 
ice water. If the flask did not break, it was heated to a higher temperature and again suddenly chilled. This was repeated with increments of $25^{\circ} \mathrm{C}$ until the flask cracked. Early in the tests it was noted that the average breaking temperature was higher for flasks which had been carefully removed from their wrappings and tested directly than it was for flasks which had been handled somewhat, in about the way glassware is commonly handled from the time it leaves the storeroom shelf until it has been cleaned and otherwise made ready for its first service in the laboratory. This handling involved relatively gentle contact of the flasks with one another and with the laboratory bench, which undoubtedly causes minute surface injuries, usually too slight to be visible. Accordingly, tests were made of two groups of 18 flasks of each brand (except Vycor). One group was tested directly as removed from the shipping containers and the other after the normal handling already described. The results are given in table 8 . The results obtained with group $A$, flasks taken directly from their shipping containers, are probably of less significance than the others, since they represent ware in a condition which cannot be maintained in service, and since they may reflect differences in the care with which the flasks were packed for shipment. On the other hand, the results of group $B$ cannot be safely regarded as representing the average condition of ware in service, since no attempt was made to learn whether the average breaking temperature decreased with prolonged or rougher handling.

TABLE 8.-Resistance to thermal shock

\begin{tabular}{|c|c|c|c|c|c|c|}
\hline \multirow{3}{*}{ Temperature } & \multicolumn{6}{|c|}{ Number of breaks } \\
\hline & \multicolumn{2}{|c|}{ Glasbake } & \multicolumn{2}{|c|}{ Kimble } & \multicolumn{2}{|c|}{ Pyrex } \\
\hline & Group $A$ & Group $B$ & Group $A$ & Group $B$ & Group $A$ & Group $B$ \\
\hline $\begin{array}{l}{ }^{\circ} C \\
175 \\
200 \\
225 \\
250 \\
275 \\
300 \\
325\end{array}$ & $\begin{array}{r}0 \\
3 \\
3 \\
4 \\
5 \\
3 \\
\end{array}$ & $\begin{array}{r}1 \\
4 \\
6 \\
5 \\
2 \\
\\
\end{array}$ & $\begin{array}{r}0 \\
2 \\
6 \\
7 \\
3 \\
\\
\end{array}$ & $\begin{array}{r}5 \\
7 \\
2 \\
2 \\
2 \\
\end{array}$ & $\begin{array}{r}0 \\
0 \\
0 \\
2 \\
3 \\
10 \\
3\end{array}$ & $\begin{array}{l}0 \\
0 \\
4 \\
6 \\
2 \\
4 \\
2\end{array}$ \\
\hline $\begin{array}{l}\text { Average breaking tem- } \\
\text { perature }\end{array}$ & 253 & 230 & 240 & 210 & 294 & 267 \\
\hline
\end{tabular}

- The relatively large number of this group which broke at $175^{\circ}$ suggested that a lower starting temperature should have been used. Accordingly, another set of 18 flasks was tested under the same conditions except with a starting temperature of $125^{\circ} \mathrm{C}$. None of this group broke at $125^{\circ}$ or $150^{\circ}$, and the average breaking temperature was $238^{\circ} \mathrm{C}$.

Vycor ware was not included in these tests. Its very great resistance to thermal shock was demonstrated by heating a flask to redness and cooling it under a water tap, without its breaking.

Washington, April 4, 1941. 